\title{
A novel engine mount with semi-active dry friction damping
}

\author{
M. Lorenz ${ }^{\mathrm{a}, *}$, B. Heimann ${ }^{\mathrm{a}}$ and V. Härtel ${ }^{\mathrm{b}}$ \\ ${ }^{\mathrm{a}}$ Mechatronik-Zentrum, Universität Hannover, Hannover, Germany \\ ${ }^{\mathrm{b}}$ Strategische Technologie, Continental AG, Hannover, Hannover, Germany
}

\begin{abstract}
In this paper the authors present a semi-active engine mount with a controllable friction damper. The normal force of the friction contact is applied by an electromagnetic actuator and can be varied dynamically. The nonlinear current-force-relation of the actuator is linearized. To account for wear and assembly tolerances, an initialization method is developed, that is based on indirect measurement of the actuators inductance. The friction contact is made up of industrial friction pads and a friction rod of steel. The friction model used is suitable especially for small oscillations of the friction damper. The control policy imitates viscous damping forces that exert a minimum of harmonics. Damping is activated only when necessary. Finally the friction mount is compared to the original mount in a row of test rack experiments and also in the car.
\end{abstract}

Keywords: Engine mount, friction damping, semi-active, reluctance actuator, friction model

\section{Introduction}

In a passenger car the engine is usually attached to the chassis via four elastic supports. Their task is to isolate the chassis from high-frequency vibrations $(24-400 \mathrm{~Hz})$ induced by the engine. At the same time high damping is necessary to minimize the low frequency engine oscillations at the eigenfrequency of the engine around $8 \mathrm{~Hz}$. These are contradicting requirements which can not be achieved without compromises through a passive mount.

In this paper a semi-active engine mount with a controllable friction damper is presented. To the best of our knowledge all previous work on semi-active engine mounts is based on hydraulic damping mechanisms [1,2] or magneto- and electrorheological fluids [3,5]. Since the first paper on semi-active friction damping in 1992 [6] there have been a number of publications on friction damped joint connections [7], car dampers [8,9] or earthquake absorbers [10] but this is the first time that dry friction is used in an engine mount. The advantage of a friction damper over a hydraulic damper in this application is two-fold: damping is limited to a maximum value which means that a friction damper does not block in case of shock excitation and the damping function can be completely switched of. This largely improves the high frequency isolation capabilities of the mount in situations where no damping is needed.

For the semi-active friction mount, the control policy employed, imitates a viscous damper with continuously variable damping. A switching logic activates the damper, whenever low frequency engine oscillations reach a defined magnitude. Under good road conditions (e.g. at a low excitation level) the engine mount exhibits soft spring behavior without any additional damping and therefore performs better than a classical mount. A specially developed electromagnetic actuator produces the necessary normal force for the friction contact. It exploits the reluctance force in the actuator's iron core. In order to save weight, this actuator operates close to magnetic saturation of the iron core thus creating a strongly nonlinear current-force characteristic which is compensated for by an intelligent control approach. This can even cope with changing air gaps due to wear of the friction pads. An initialization

\footnotetext{
*Corresponding author. E-mail: Marc-Lorenz@web.de.
} 

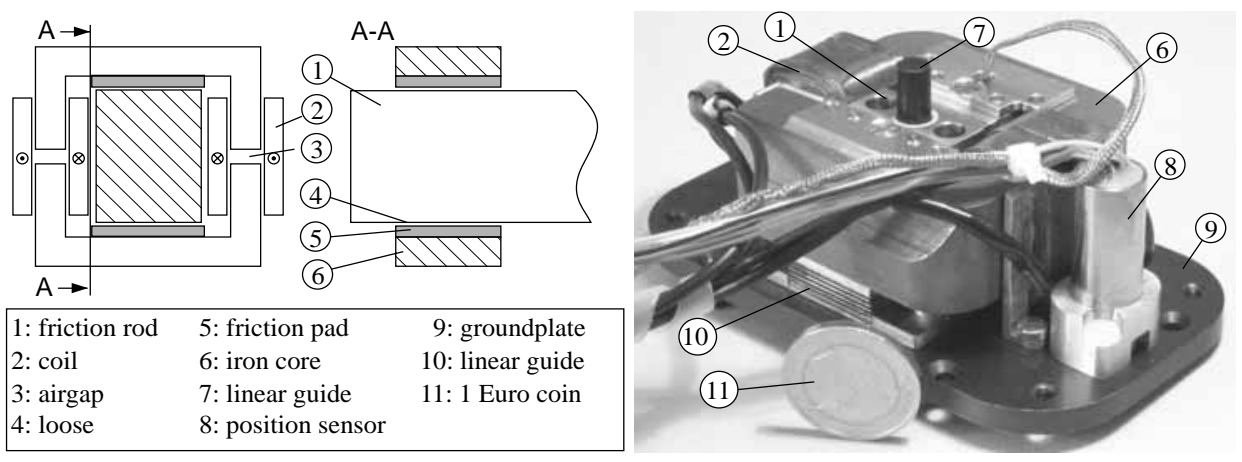

Fig. 1. Schematic of the electromagnetic actuator (left) and photo of the prototype friction damper (right).

method is developed, that calculates a valid current-force-relation each time the actuator is switched on. It calculates the inductivity of the actuator from the small current ripples that are caused by the switching power amplifier. For optimum performance an appropriate model of the friction contact is necessary that includes nonlinear dynamic behavior. In especially designed experiments these effects are assessed and an appropriate dynamic friction model is derived.

The presented prototype fully replaces the original right-hand side engine mount of a 2.01 Diesel engine in a test-vehicle. Experimental evaluation carried out on the test-rack and in the car confirmed the superior performance of the friction damping approach over the original passive mount.

\section{Prototype}

The original mount of the test-vehicle is a passive hydro mount which consists of an elastomer spring and a hydraulic damping mechanism. The hydraulic damper was replaced by the friction device, while the housing and the elastomer spring were taken from the original mount. The modified mount is of the same size as the original one with an additional weight of $200 \mathrm{~g}$. Figure 1 shows the friction damper unit of the mount. The electromagnetic actuator creating the normal force for the friction contact consists of a toroidal iron core, which is divided into two halves by two air gaps. Figure 1 shows the scheme of the actuator. The friction contact consists of two ordinary friction pads from industrial brakes which are pressed against a moving friction rod by the actuator. In the engine mount the friction rod is connected to the engine bracket and the ground plate with the actuator is connected to the chassis. The force of the actuator is given by the reluctance force between the two halves of the iron core. These halves are attached to a ground plate via a combination of leaf springs and elastomer elements, providing a very high stiffness in the direction of the friction force and at the same time a high compliance for the horizontal movement of the actuator which is necessary to bring the friction pads in contact with the friction rod. The whole unit is very small (see the one Euro coin in Fig. 1).

\section{Electromagnetic actuator}

Precise control of the friction normal force is essential for the whole concept. In the following section an effective control method for the reluctance force actuator is presented that does not need any separate force or displacement sensor.

For a reluctance force actuator the force $F$ is determined by the flux density $B$ and the area $A$ of the air gap:

$$
F=\frac{A}{2 \cdot \mu_{0}} \cdot B^{2}
$$

The relation between the coil current $i$ and flux density $B$ is given by 


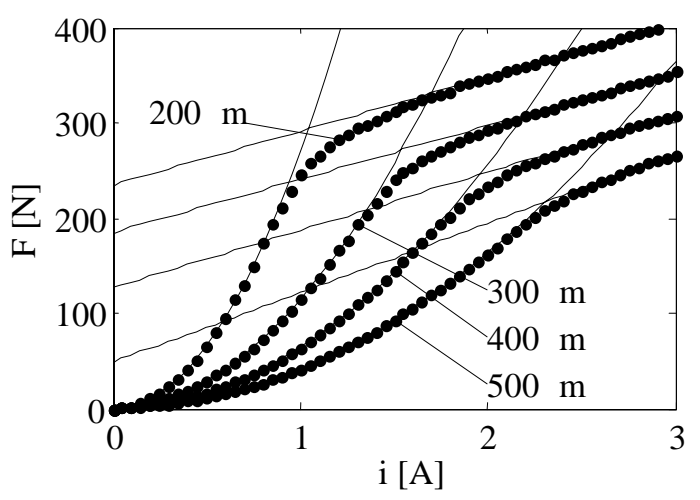

Fig. 2. Current-force relation for different distances between the iron core halfs.

$$
B=n \cdot i \cdot\left(\frac{2 \delta}{\mu_{0}}+\frac{l_{F e}}{\mu_{0} \cdot \mu_{r}}\right)
$$

where $n$ is the number of windings of the coil, $\delta$ is the distance between the two halves of the iron core and $l_{F e}$ is the total length of the iron core. For small flux densities the permeability $\mu_{r}$ of the iron is much bigger than the permeability of air $\mu_{0}$, so that the influence of the iron on the flux density and on the reluctance force can be neglected. In this case force is a quadratic function of the coil current i. In order to realize a relatively lightweight actuator, the iron core has to be operated close to magnetic saturation (For the material used this is approximately $B=1.4 \mathrm{~T}$ ). In this region the relative permeability $\mu_{r}$ of the iron drops significantly with increasing flux density. The dots in Fig. 2 represent current-force-characteristics measured at different air gaps. Because of tolerances in the assembly, the initial gap width is not known and the gap becomes smaller with increasing wear. From the measured data it is obvious that the influence of the air gap can not be neglected. The direct measurement of the actuator force through a force sensor is expensive and makes the construction more complex. Since the gap width varies very slowly and can be assumed to be constant over one driving cycle we used a mapped lookup table for determination of the force. The practical use of the lookup table presented two main problems. Firstly the characteristics have to be represented by an algebraic function in order to reduce the amount of data necessary. Secondly the air gap should be determined without using a separate sensor, because such sensor would be of no principal advantage over a force sensor.

\subsection{Parametrization of the lookup table}

As expected from Eqs (1) and (2) the first part of the function can be approximated by a second order parabola. Towards higher currents it can be approximated by a linear function which is an effect of the steep drop of the permeability of iron in that area. These two facts were used as a starting-point to generate the fitting function.

$$
\begin{aligned}
F(i, \delta)= & \left(a_{1}(\delta)+a_{2}(\delta) \cdot i\right) \cdot\left[\arctan \left(a_{3}(\delta) a_{4}(\delta)\right)+\arctan \left(a_{3}(\delta)\left(i-a_{4}(\delta)\right)\right)\right] \\
& +a_{5}(\delta) \cdot i^{2} \cdot\left[\arctan \left(a_{3}(\delta) a_{4}(\delta)\right)-\arctan \left(a_{3}(\delta)\left(i-a_{4}(\delta)\right)\right)\right]
\end{aligned}
$$

where $a_{1}$ and $a_{2}$ are the parameters of the linear function and $a_{5}$ is the parameter of the parabola. Parameters $a_{3}$ and $a_{4}$ determine the transition from the quadratic to the linear part of the curves. All parameters $a_{i}$ are functions of the distance $\delta$ between the halves. The parameters $a_{i}$ were identified by a standard nonlinear optimization routine under Matlab for different constant values of the gap width $\delta$. The change of these parameters with increasing air gap can be modeled by polynoms of the form

$$
a_{i}(\delta)=\sum_{n} p_{n} \delta^{n-1}
$$

The parameters identified for the electromagnet in the friction damper are summed up in Table 1. The necessary amount of data is reduced from a large lookup table to a single nonlinear function with 18 parameters. 
Table 1

Identified parameters of the nonlinear function

(Eqs (3) and (4)) for fitting the measured data

\begin{tabular}{ccccc}
\hline & $p_{1}$ & $p_{2}$ & $p_{3}$ & $p_{4}$ \\
\hline$a_{1}$ & 70.2 & -0.157 & $5.84 \mathrm{e}-4$ & $-9.77 \mathrm{e}-7$ \\
$a_{2}$ & 77.2 & -0.305 & $5.28 \mathrm{e}-4$ & $-2.58 \mathrm{e}-7$ \\
$a_{3}$ & 20.7 & -0.11 & $2.3 \mathrm{e}-4$ & $-1.59 \mathrm{e}-7$ \\
$a_{4}$ & $-8.9 \mathrm{e}-3$ & $4.6 \mathrm{e}-3$ & - & - \\
$a_{5}$ & 354 & -2.21 & $5 \mathrm{e}-3$ & $-3.85 \mathrm{e}-6$ \\
\hline
\end{tabular}

\subsection{Indirect measurement of the air gap}

With the parameterization given above, the actual current-force relation is calculated at the beginning of each new driving-cycle. The crucial input parameter for this function is the gap width between the two iron core halves. This value is determined by an indirect method that exploits the high frequency switching effects of the power amplifier which consisted of a four quadrant H-bridge of MOSFETs (the standard form of power amplifier for inductive loads). These amplifiers change the polarity of voltage across the coil at frequencies in the $\mathrm{kHz}$ region. Current is controlled by changing the time-relation between the two switching states, the so called duty cycle. At a duty cycle of $50 \%$, i.e. when positive and negative voltage are applied for equal times, the mean current is $0 \mathrm{~A}$ overlaid by small current-ripples that are caused by the fast changes in polarity. Each of those ripples can be regarded as a step response (input is the voltage step, output is the current) which produces small changes in the actuator's magnetic field. The rate of change of the flux density $B$ in the iron core is measured with a separate coil whereas the change rate of the coil current is measured with a high frequency transformer. Both values are measured simultaneously $18 \mu \mathrm{s}$ after switching. From these two values the incremental inductance

$$
L_{i}=\frac{d i / d t}{d B / d t}=\frac{d i}{d B}
$$

is calculated. Note that it is not possible to calculate the width of the air gap directly from this inductance, as one might expect. The reason is the ambiguous relation between the incremental inductance, the current and the distance in question.

The total inductance $L$, which is easily calculated as the integral of $L_{i}$ over the current $i$

$$
L(i, \delta)=\int_{0}^{i} L_{i} d i
$$

should, for a given system, theoretically be unambiguously related to the actual current and the air. However for our system this is not the case because the high frequency current-ripples only influence the magnetic field close to the surface of the iron core (skin effect). This is due to the relatively slow diffusion of the magnetic field into the iron in the region of $\mu \mathrm{m}$ per $\mu \mathrm{s}$. The diffusion rate is inversely proportional to the permeability of the iron and its conductivity. As explained above, permeability decreases as saturation occurs. This results in a much faster diffusion, so that the amount of material that is affected by the changing magnetic field increases with increasing current. Finally it can be said that it is not possible to obtain a quantitatively correct value of either total or incremental inductance from the current-ripples of a reluctance actuator switched at high frequencies. To give the reader a qualitative understanding of this effect, results of an FEM-simulation are given in Fig. 3. The upper left diagram shows the total magnetic field in the area of one of the two air gaps of the actuator at a current of $3 \mathrm{~A}$ (maximum force). In the upper right diagram the alternating part of the magnetic field is depicted. Flux density along the section depicted in the upper right diagram is given in the lower left and right diagrams for different mean currents. It can be seen that the propagation of the magnetic field is much faster in case of the higher current.

Because of the problems mentioned above the incremental inductance can only be taken as a measure for the air gap in regions were the relation $L_{i}(i, \delta)$ is unique over the whole current range of the actuator from 0 to $3 \mathrm{~A}$. In the left diagram of Fig. 4, measured values of the incremental inductance for constant gap widths as defined in Eq. (5), are depicted. Two sections of this diagram are defined. First, the inductance as a function of the gap width at a current of $0.2 \mathrm{~A}$ which is called $L_{0.2}(\delta)$ and second current as a function of the gap width at a constant inductance 


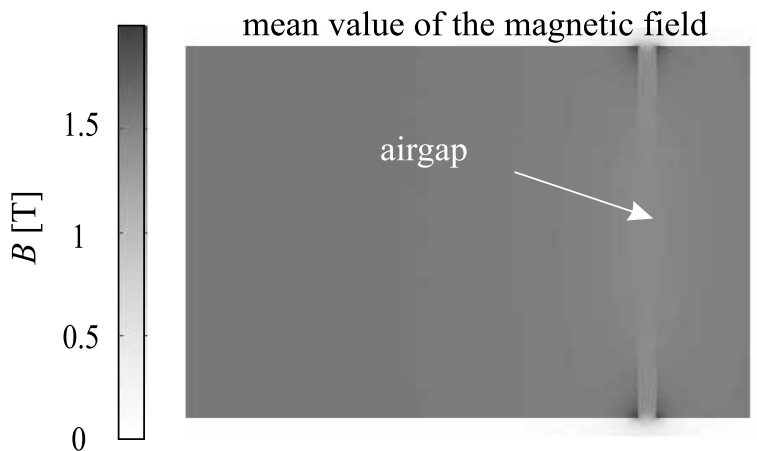

field propagation at $3 \mathrm{~A}$ mean current

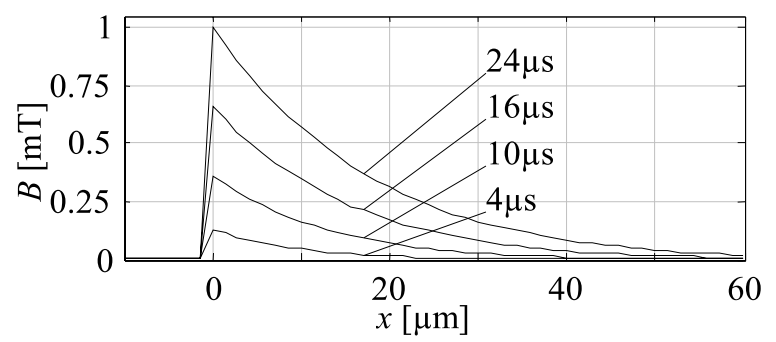

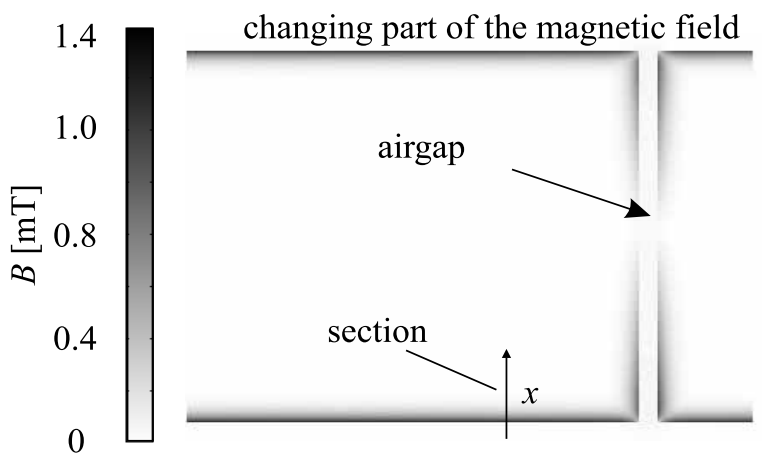

field propagation at $1 \mathrm{~A}$ mean current

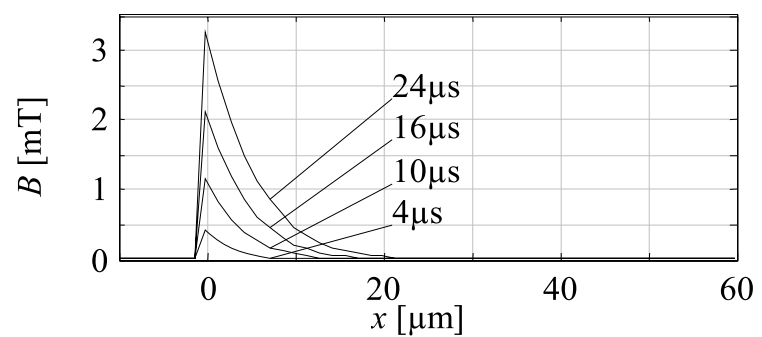

Fig. 3. Results of an FEM simulation of the magnetic field at high frequency switching of the coil-voltage.
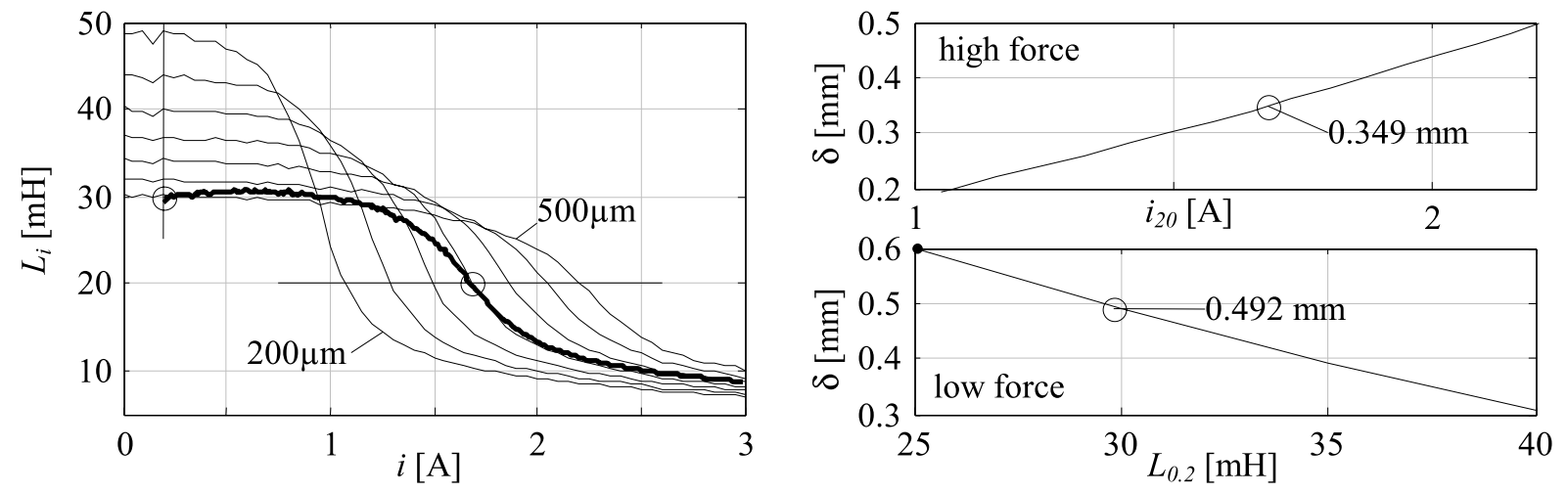

Fig. 4. Measurement of the distance between the two halves of the iron core. Left: measured value of the incremental inductance at different constant gap widths (slim) and under real conditions at the assembled friction damper (wide). Right: Sections througt the diagram on the left for constant inductance of $20 \mathrm{mH}$ (upper diagram) and for constant current of $0.2 \mathrm{~A}$ (lower diagram).

of $20 \mathrm{mH}$ which is called $i_{20}(\delta)$. These two functions are depicted in the right part of Fig. 4. The circles represent measured data, the curve belongs to a polynom approximation. This means, the gap width can be measured at low current, when the actuator force is very small and the halves of the iron core are in rest position (with an air gap between friction pads and friction rod) and at high current, where the friction pads are pressed against the friction rod. In the left diagram of Fig. 4, measured incremental inductance taken from a current ramp up at the assembled friction damper is given as whide line. It can be seen that at low current the distance of the iron core halves is $492 \mu \mathrm{m}$ and, as current increases the gap decreases to $349 \mu \mathrm{m}$. From the difference of $153 \mu \mathrm{m}$, an offset-current can be calculated that brings the friction pads into a ready-position with light pressure against the friction rod. The actual current-force relation is calculated for the measured gap width of $349 \mu \mathrm{m}$ in this case. This leads to an initialization routine that is executed each time the damper is switched on. The flow-diagram of the initialization routine is given in Fig. 5. 


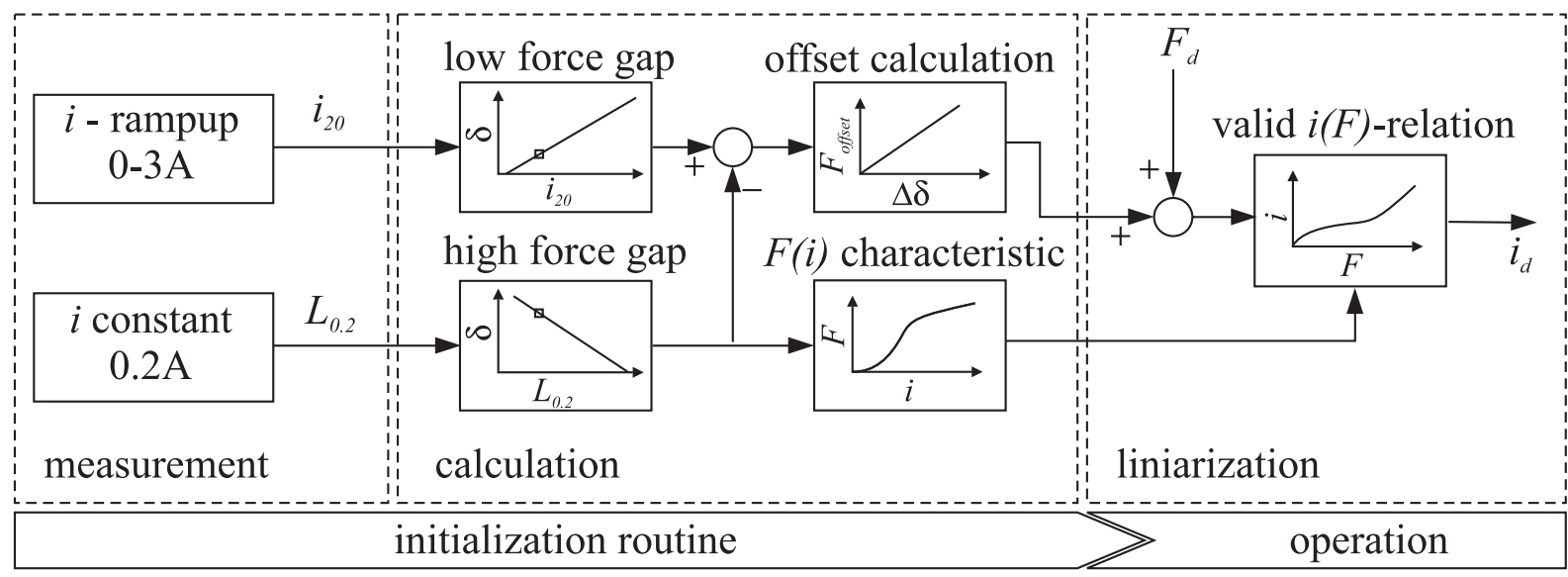

Fig. 5. Scheme of the proposed indirect method to find a valid force-current-relation for the linearization of the electromagnetic actuator.
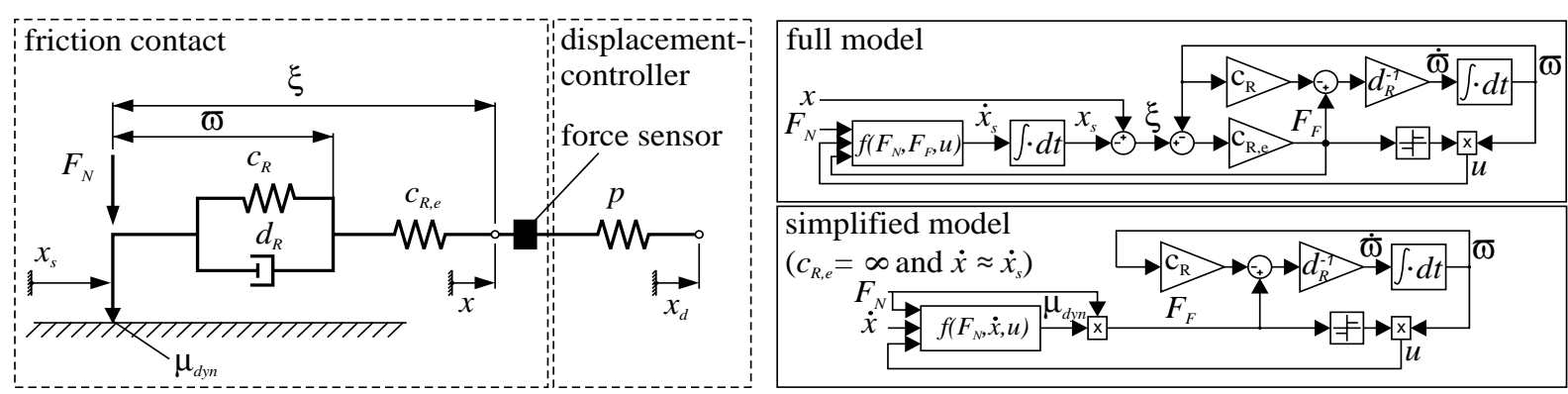

Fig. 6. Special elastoplastic friction model used for modeling the nonlinear friction effects of the friction interface between industrial brakes material and stainless steel.

\section{Friction model}

The aim is to directly control the friction force which is the damping force of the engine mount. As first part of this control the initialization process of the normal force actuator reveals a good linearization of its current-forcecharacteristic. Now secondly, the dynamic behavior of the friction contact has to be taken into account. From literature a number of dynamic friction models are known, from the Dahl Model to the LuGre model. These models prove to be perfectly suited for lubricated friction interfaces between metal surfaces. In case of the friction damper used, the friction contact consists of stainless steel and friction pads of standard metal free brakes material on the basis of synthetic resin. By experimental investigation it was shown, that the existing friction models are difficult to identify for our friction contact because they assume a stribeck effect, i.e. a drop in friction force with increasing relative sliding velocity in the region of low speeds. Our friction contact on the contrary does not exhibit this effect. A number of different brakes materials were tested and only those that contain metal show a neglibly small stribeck effect. Instead, other effects as for example shear elasticity and damping of the friction pad play a greater role. So finally we developed a friction model that represents the dynamic behavior especially for oscillating motion of a friction contact with brakes material. It is easy to identify as it has a simple schematic representation. In the following, the model is presented and the identification process is described.

In the left part of Fig. 6, the mechanical model of the friction contact is depicted. Tangential stiffness and damping of the friction interface are represented by the linear spring damper elementc $c_{R}, d_{R}$ and $c_{R, e}$. The nonlinear part of the friction model is concentrated at the sliding interface, where a dynamic friction coefficient $\mu_{d y n}$ is defined. When the friction contact is moved from rest along the coordinate $x$, the sliding interface coordinate $x_{s}$ remains constant for small displacements $x$ while an elastic deformation occurs at the spring damper element. This is used later to identify the linear parameters. At a certain displacement or force-level, the sliding interface begins to move. 

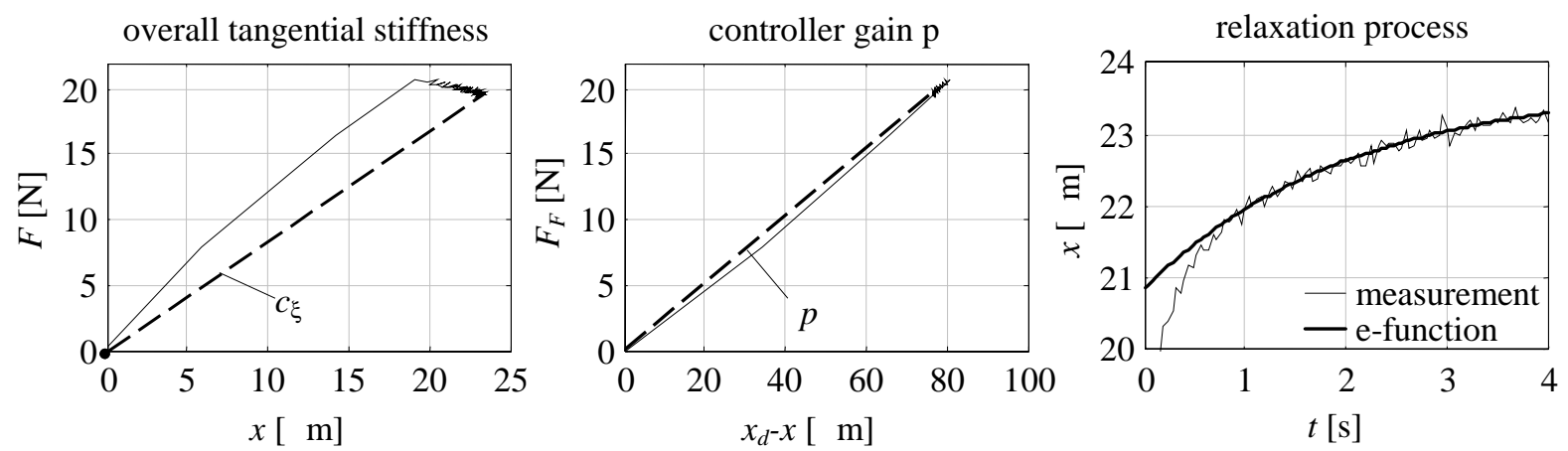

Fig. 7. Step 1 of the identification process for the proposed friction model.

In experiments it was observed that the force level in sliding, i.e. the dynamic friction coefficient, is dependant on the strain $\varpi$ of the linear element. The larger the strain $\varpi$ in sliding direction, the lower the friction force. As measure for this relative strain, the variable $u$ is introduced, which is defined as

$$
u=\operatorname{sign}\left(F_{F}\right) \cdot \varpi
$$

After a change in direction, i.e. after a change of the sign of the friction force $F_{F}$, the dynamic friction coefficient $\mu_{\text {dyn }}$ jumps to a higher value because $u$ becomes bigger. As the friction contact is moved in the opposite direction, it decays to lower values again. A block-diagram representation of the model described by Eqs (7) and (8) is given in the upper right part of Fig. 6. This will be referred to as "full model". It has the form

$$
\begin{aligned}
F_{F} & =c_{R, e}\left(x-x_{\text {gleit }}-\varpi\right) \\
\dot{x}_{s} & =f\left(F_{F}, u, F_{N}\right) \\
\dot{\varpi} & =d_{R}^{-1}\left(F_{F}-c_{R} \varpi\right)
\end{aligned}
$$

In simulations, this model turned out to be very precise especially in the region of changes in the sliding direction. Unfortunately it requires very short time steps because the spring $c_{R, e}$ in combination with the sliding interface causes numerical problems. Therefore, the full model is not suited for implementation in a real time precontrol. Instead a simplified model was defined that omits the spring $c_{R, e}$. A significant difference between the sliding velocity $d \varpi / d t$ and the outer measurable velocity $d x / d t$ only occurs at turning-points of the oscillating motion. If this is not an important state, the model can be further simplified by defining $d x / d t \approx d \varpi / d t$. Although the simplified model is imprecise at direction changes, it is suficient for the application described here. The model equation is

$$
\begin{aligned}
\mu_{d y n} & =f\left(F_{N}, \dot{x}, u\right) \\
\dot{\varpi} & =d_{R}^{-1}\left(\mu_{d y n} F_{F}-c_{R} \varpi\right)
\end{aligned}
$$

This simplified model is given in the block-diagram in the lower right part of Fig. 6 . In the following the identification process for the proposed model is described. First, the full model will be identified, than it will be simplified. Finally both models are compared. The experimental setup that was used, consists of a linear motor that can apply arbitrary desired displacements $x_{d}$ to the friction contact. The actual displacement $x$ and the friction force $F_{F}$ can be measured. Position control of the linear motor is implemented via a simple proportional controller that is represented by spring $p$ in the left diagram of Fig. 6. In a first step, the linear part of the model is identified. Therefore, a constant normal force is applied and the desired displacement is ramped up fast and then held constant. The whole process takes place in the microslip domain, i.e. no sliding $x_{s}$ occurs. During the ramp up of $x_{d}$ the major part of the strain occures at springs $p$ and $c_{R, e}$, because of the relatively large time constant of the spring damper element $d_{R}, c_{R}$. After the ramp, at constant $x_{d}$, relaxation of this spring damper element can be observed.

The left diagram in Fig. 7 shows a measured displacement-force-curve for the described experiment. After relaxation of the damper, the joint stiffness $c_{\xi}$ of the springs $c_{R, e}, c_{e}$ and $p$ can be obtained as the relation between the measured displacement and the measured force level as depicted in the left diagram. In the middle diagram of the 
Table 2

Identified parameters of Eqs (13) and (14)

\begin{tabular}{llll}
\hline$b_{\text {offset }}: 14 \mathrm{~N}$ & $\beta_{2}: 8.09 \mathrm{e}-3 \mathrm{~N}^{-1}$ & $a_{p, 2}: 0.1781 \mathrm{~s} / \mathrm{mm}$ & $\alpha_{p, 1}: 0.5829$ \\
\hline$b_{1}:-18.51 / \mathrm{mm}^{2}$ & $a_{n, 1}: 0.802$ & $a_{n, 3}: 0.4177$ & $\alpha_{n, 2}: 0.0032 \mathrm{~N}^{-1}$ \\
$b_{2}:-1.7 \mathrm{~s} / \mathrm{mm}^{2}$ & $a_{p, 1}: 0.5829$ & $a_{p, 3}: 0.118$ & $\alpha_{p, 2}: 0.004 \mathrm{~N}^{-1}$ \\
$\beta_{1}: 0.174$ & $a_{n, 2}: 0.3253 \mathrm{~s} / \mathrm{mm}$ & $\alpha_{n, 1}: 0.688$ & \\
\hline
\end{tabular}
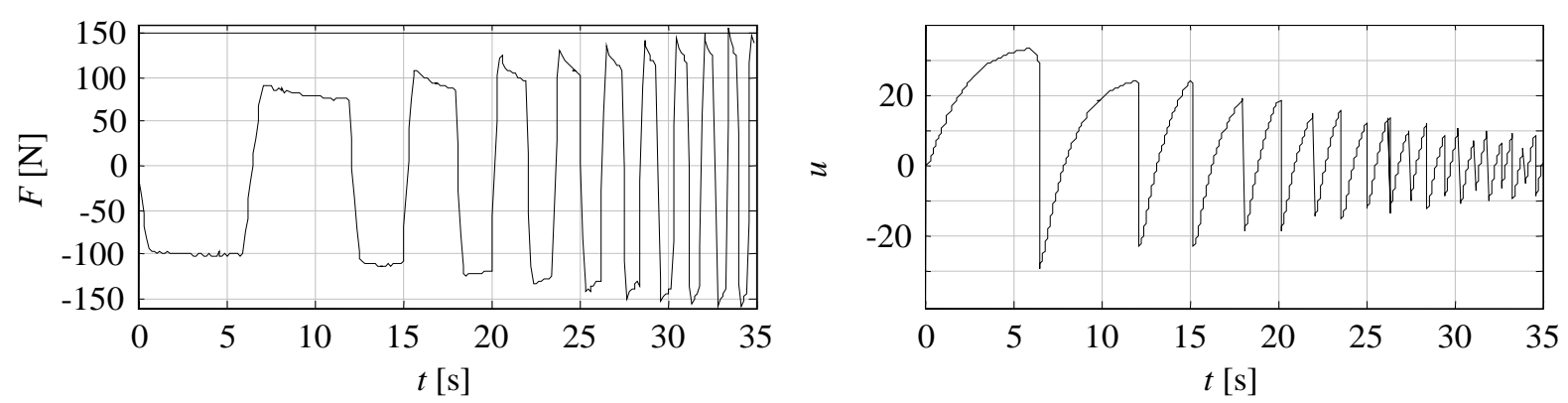

Fig. 8. Simulation of the relative deflection $u$ (right) with the measured force (left).

same figure, the measured force $F_{F}$ is plotted over the difference between desired and measured displacement. With this, the correct value of the spring constant $p$ can be determined. Finally the measured displacement $x$ is plotted over time in the right diagram of Fig. 7. Here the time constant $T$ and the asymptotic value $k$ of the displacement can be easily determined by fitting the exponential function

$$
x(t)=k\left(1-\frac{T_{v}}{T} e^{-\frac{t}{T}}\right)
$$

to the measured curve. Good congruence of the measured and the fitted curve can be seen for times greater than $1 \mathrm{~s}$. This is because the first part of the measured curve is influenced by the ramp-up. For an ideal step input of $x_{d}$, the curves should be congruent at all times. The searched linear parameters can be calculated from the measured values of $c_{\xi}, p$ and $T$ as

$$
c_{R, e}=\frac{k \cdot c_{\xi} \cdot p}{p-k \cdot c_{\xi}} ; \quad c_{R}=\frac{c_{\xi} \cdot c_{R, e}}{c_{R, e}-c_{\xi}} ; \quad d_{R}=c_{R} \cdot T
$$

After the linear part is identified, the relation between the relative deflection $u$ and the dynamic friction coefficient $\mu_{d y n}$ is examined, which is step two of the identification process. For this the friction contact is moved oscillatory with constant velocity between two turning points. After every second turning point the velocity is incremented by $1 \mathrm{~mm} / \mathrm{s}$. The measured force that is depicted in Fig. 8 is used as input to a simulation of the linear part. This reveals the corresponding displacement $u$ which can also be seen in Fig. 8 .

As mentioned before, this relative deflection $u$ is proportional to the drop in friction force after a turning point. This effect can be clearly seen in Fig. 9, where the friction coefficient is plotted over the deflection $u$ for constant velocities. While the time curve of the measured force has an exponential characteristic in the region of constant velocities, straight lines are derived when $\mu$ is plotted over $u$. These lines represent the relation between the deflection $\mathrm{u}$, the velocity $d x / d t$ and the dynamic friction coefficient $\mu_{d y n}$ at constant normal force $F_{N}$ (Eq. (9)). In the following third step of the identification this measurement is conducted for different constant values of the normal force between $40 \mathrm{~N}$ and $120 \mathrm{~N}$. For each normal force the parameters of the linear approximation

$$
\mu_{d y n}\left(F_{N}, \dot{x}_{s}, u\right)=a\left(F_{N}, \dot{x}_{s}\right)+b\left(F_{N}, \dot{x}_{s}\right) \cdot u
$$

are identified (see Fig. 9). The identified values of the parameters $a$ and $b$ are depicted as dots in Fig. 10. Here $d x / d t \approx d x s / d t$ can be assumed, because the data is measured at constant velocity where $d x / d t>>d \xi / d t$.

As an analytic approximation for parameter $b$, the relation

$$
b\left(F_{N}, \dot{x}_{s}\right)=b_{\text {off set }}+\left(b_{1}+b_{2} \cdot \dot{x}_{s}\right) \cdot\left(\beta_{1}+\beta_{2} \cdot F_{N}\right)
$$



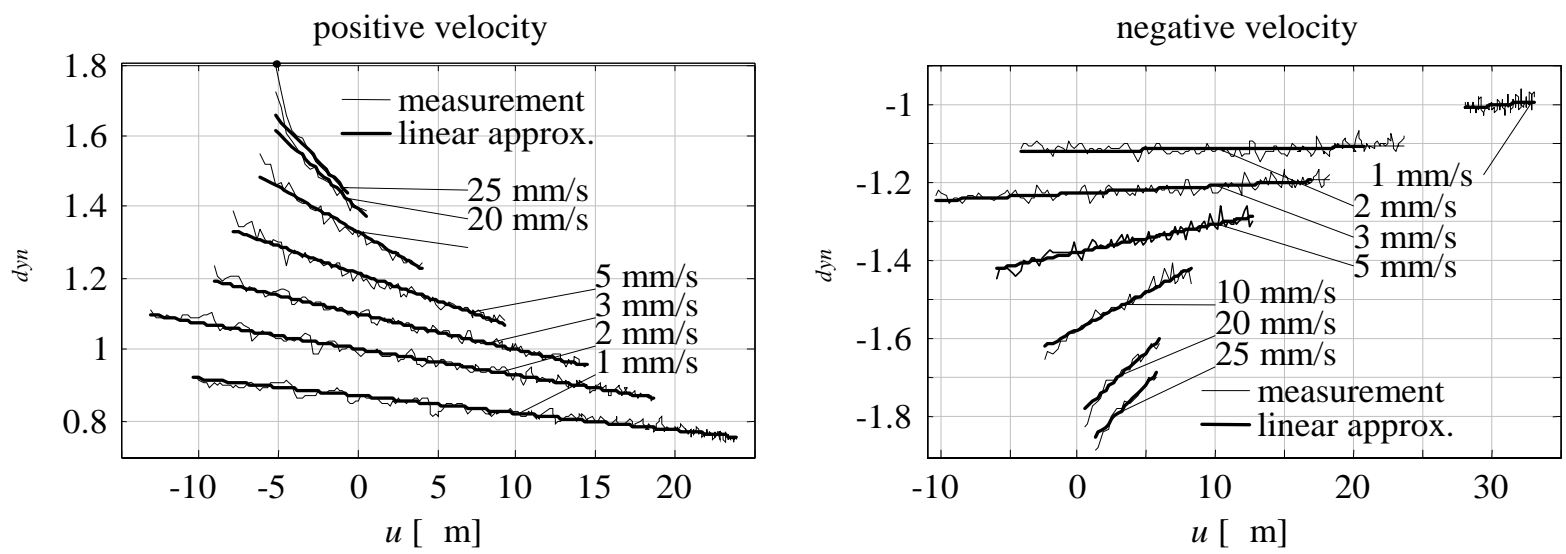

Fig. 9. Dynamic friction coefficient $\mu_{d y n}$ plotted over the deflection $u$ for different constant velocities $d x / d t$ at constant normal force of $100 \mathrm{~N}$ (derived from the measurement depicted in Fig. 8).
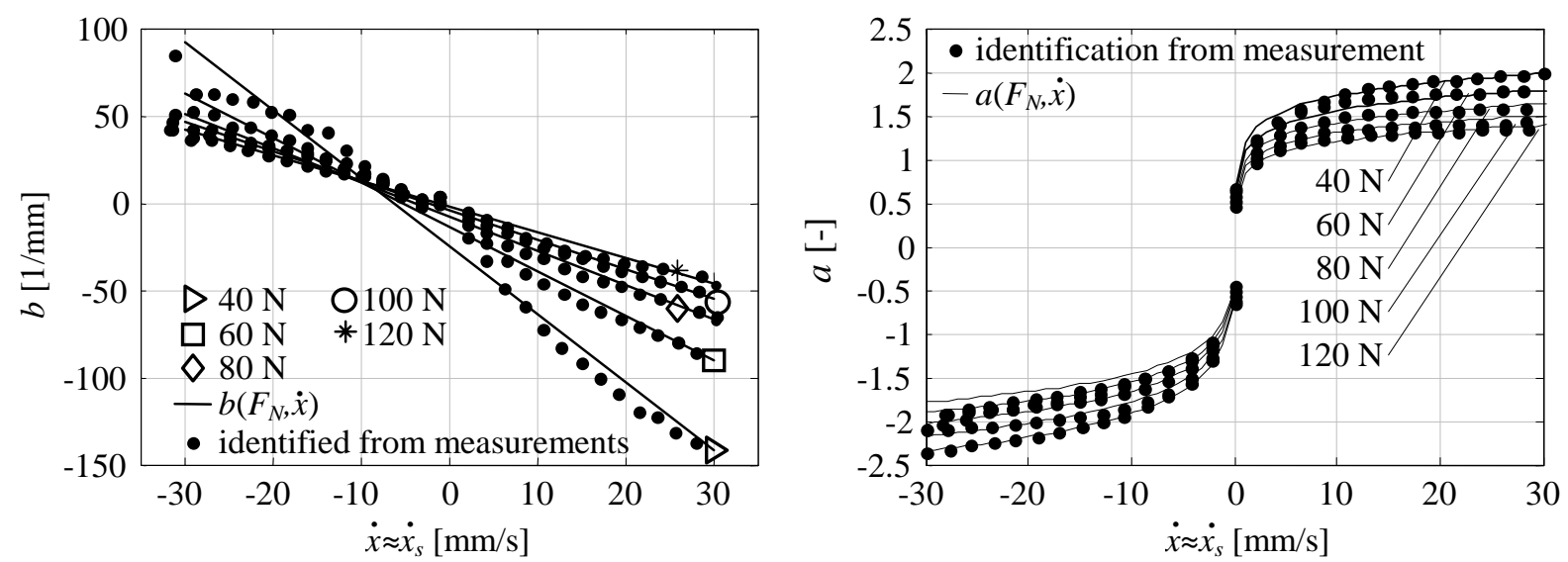

Fig. 10. Parameters $a$ (right diagram) and $b$ (left diagram) of Eq. (9). Identified values for different constant velocities and different normal forces are displayed as dots, the approximation is displayed as line.

has been found. The curves of parameter $a$ for constant normal force can be approximated by a logarithmic function of the form

$$
a\left(F_{N}, \dot{x}_{s}\right)=\left[a_{p / n, 1}+a_{p / n, 2} \cdot \ln \left(a_{p / n, 3}+\frac{\left|\dot{x}_{s}\right|}{\dot{x}_{o}}\right)\right] \cdot\left[\alpha_{p / n, 1}+\alpha_{p / n, 2} \cdot F_{N}\right] ; \quad \dot{x}_{0}=1 \frac{m m}{s} .
$$

Here separate parameters are necessary for positive (index $p$ ) and negative (index $n$ ) velocities. The parameters, identified for the friction pad used in the damper are given in Table 2. The result of this identification can be seen in Fig. 10, where the analytic approximation is depicted as a line.

The expression $\mu_{d y n}=f\left(F_{N}, d x_{s} / d t, u\right)$, which consists of Eqs (12), (13) and (14) has the correct form for the simplified friction model. However, some facts have to be regarded when using $x$ instead of $x_{s}$. At zero velocity, the expression for $\mu_{d y n}$ has two possible values and the simplified model is not defined for microslip, where $x_{s}=0$, anyway. This problem is solved by multiplying the expression with a weighting function that fades $\mu$ dyn to zero at very low velocities.

For the full model Eqs (12), (13) and (14) must be rewritten as $d x_{s} / d t=f\left(F_{N}, F_{F}, u\right)$. Because of the complexity of the nonlinear expressions, lookup tables were used for the implementation of both models on a real time processor.

For verification and comparison of both versions of the proposed model, measured displacement data from the saw tooth test-function was used as model input. In Fig. 11 the result is depicted. Both models show very good 


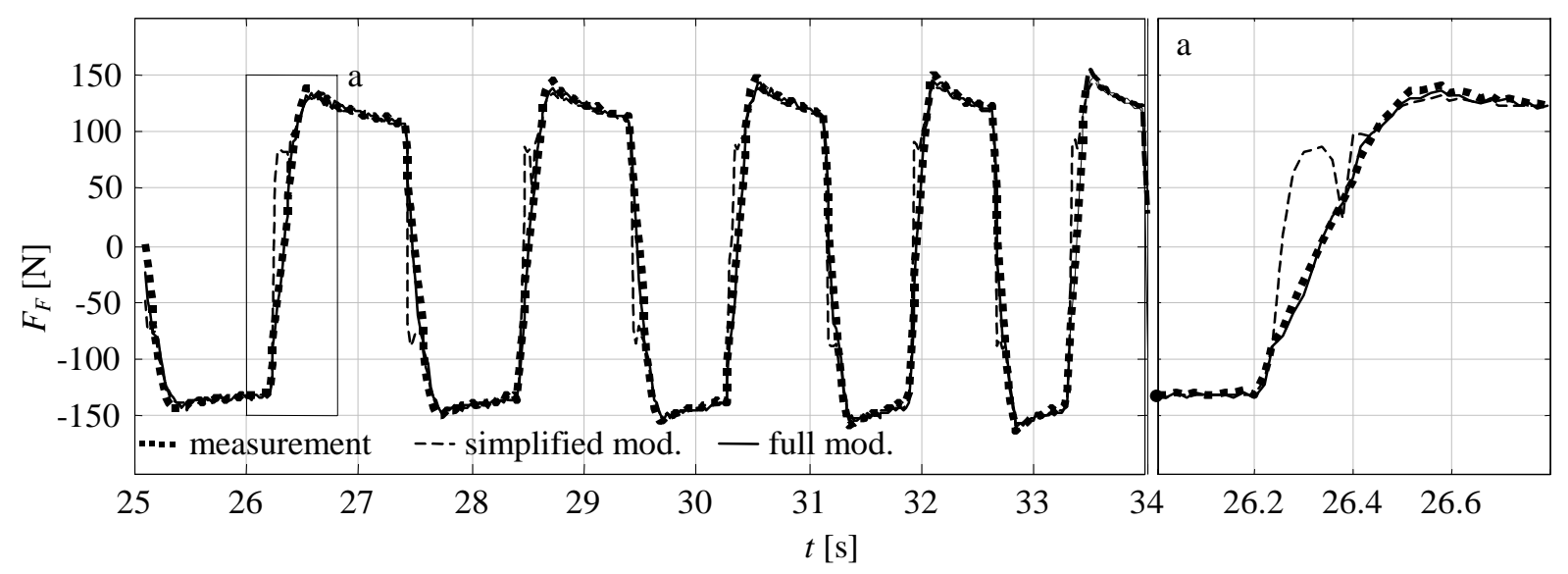

Fig. 11. Verification of the proposed friction model and comparison of the full and the simplified model.

accordance between measured and calculated data in the macroslip region. In the microslip domain the full model is very precise while the simplified model shows large deviations, as expected. The friction damper of the engine mount must not stick, because sticking leads to higher harmonics in the damper force and this can cause acoustic problems. Therefore sticking and microslip are not important and the simplified model can be used.

\section{Control}

The idea behind the control policy implemented on the friction damped mount is to apply damping only if the engines natural frequency is excited, which is typically tuned to values between 5 and $10 \mathrm{~Hz}$. Main external excitation source for this low frequency vibration is the road, but also shifting, sudden acceleration or braking can be (inner) reasons. Mostly these events have only a short duration. The vibrations that are caused by the engine itself reach from a low value of around $25 \mathrm{~Hz}$ in idle to more than $300 \mathrm{~Hz}$ at high speed. The distance in frequency domain between the two different vibration phenomena is large enough for a clear separation of the eigenfrequency by using a bandpass filter. Good vibration isolation, superior to those of passive engine mounts, can be achieved when damping is completely deactivated. Only for a very short period of time after a (transient) excitation of the eigenfrequency, damping is needed. It serves to limit the engine motion for package but also for comfort reasons because the low frequency engine vibration lies in the same frequency region as the natural vibration modes of some inner human organs.

Most intelligence of the engine mount control is concentrated in the precontrol of the friction contact and in the initialization routine of the electromagnetic actuator. The global control policy is relatively simple as visualized in Fig. 12. The velocity $d x / d t$ is obtained from the differentiated and bandpass-filtered signal of the build-in displacement sensor of the mount. The passband frequency is set to the eigenfrequency of the engine. Only the vibration at this eigenfrequency has to be damped. Its magnitude serves as criterion for on- and offswitching of the damping function, were the switch on level is chosen higher than the switch off level, to avoid oscillatory switching. The desired friction force is calculated from the filtered $d x / d t$ via a static damper characteristic (here realized as lookup table). Viscous (velocity-proportional) damping is imitated because this provides optimal acoustic behavior. Around zero velocity a dead zone is necessary to avoid sticking of the damper. The filtered $d x / d t$ is also used for a real time simulation of the simplified friction model. From this simulation, the current value of the dynamic friction coefficient is obtained at each time step which is used to calculate a desired normal force. The relation between desired normal force and actuator current is given in the lookup table, which is calculated by the initialization routine described in Section 3. 


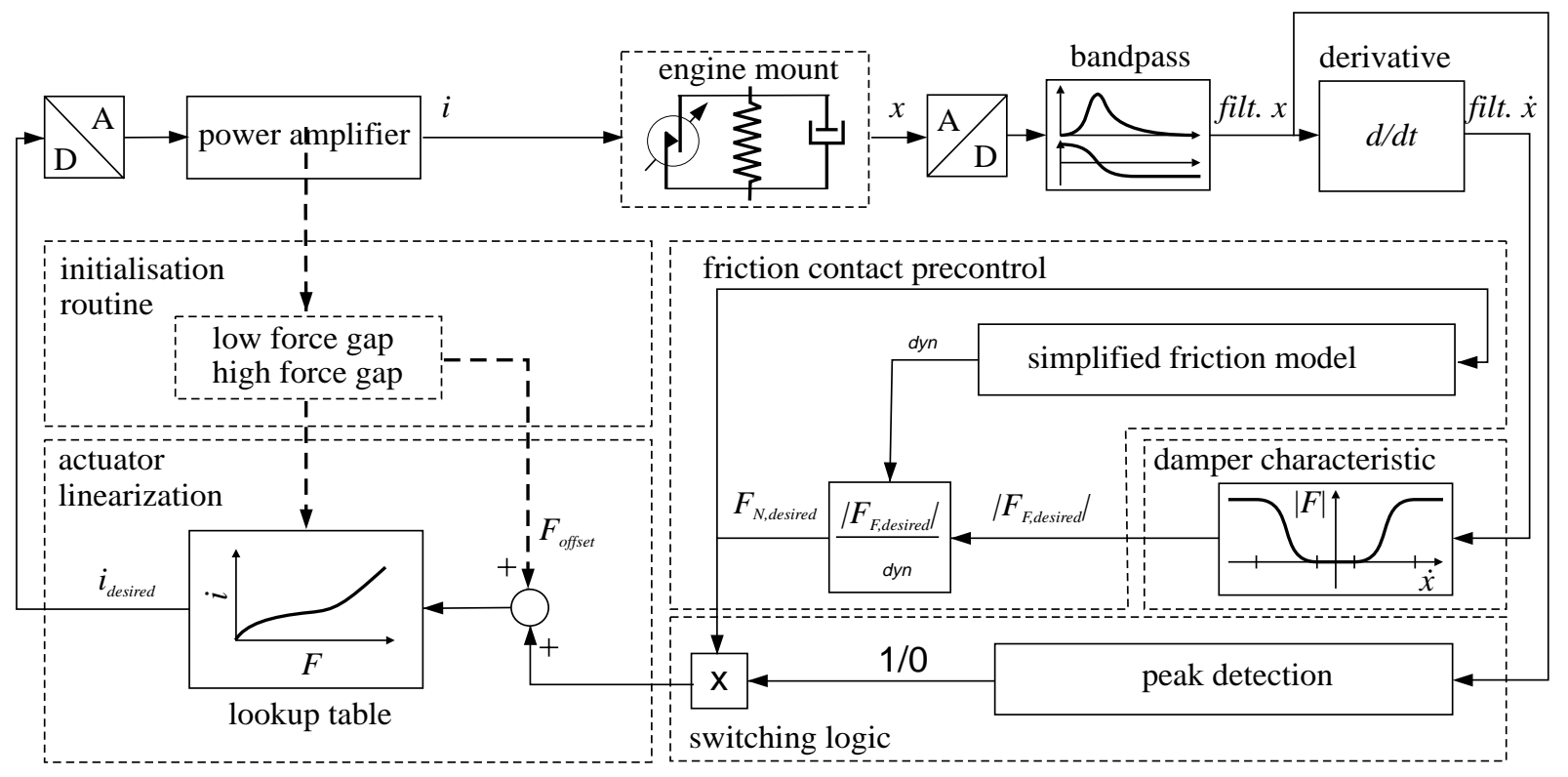

Fig. 12. Block diagram of the control implemented in the friction damped engine mount.
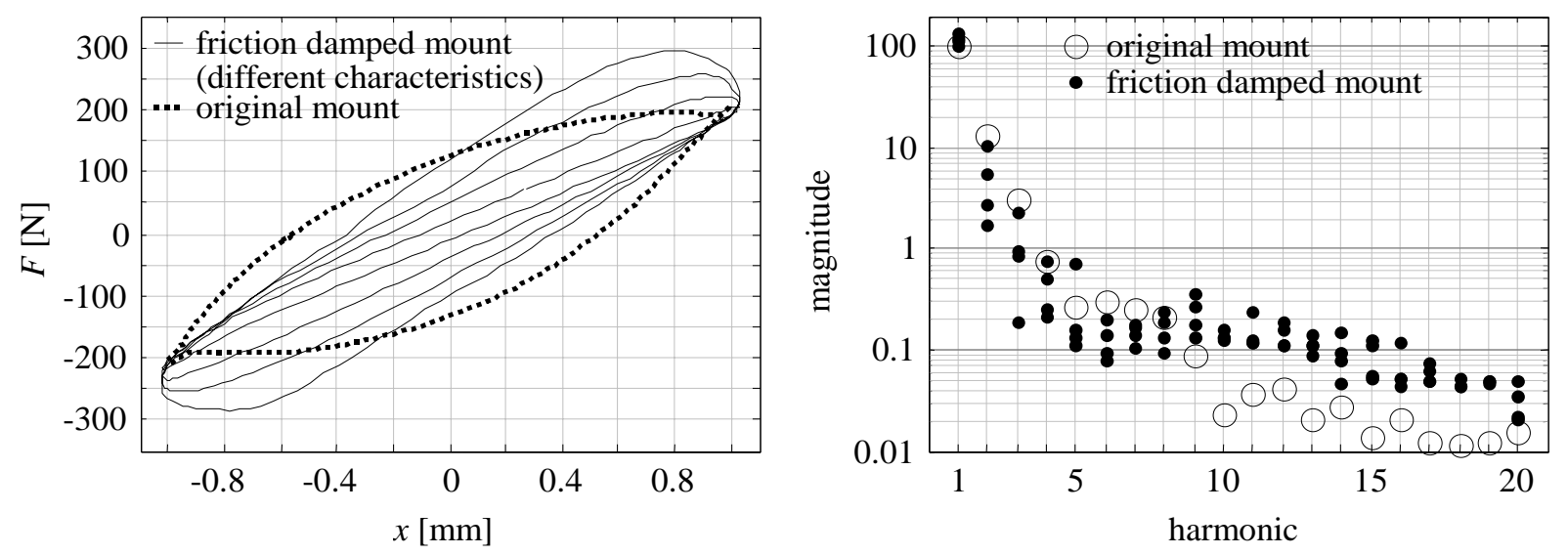

Fig. 13. Comparison of the original and the semi-active friction mount in a test-stand experiment (at sinusoidal displacement). Loss-hysteresis (left) and analysis of harmonics in the measured force.

\section{Experimental evaluation}

Before the engine mount was built into the car, it was tested in a special hydropuls-tester. This device can generate a sinusoidal displacement at preselected frequencies, which is ideal for the empirical optimization of the damper characteristic block in Fig. 12. On the left side of Fig. 13 the hysteresis of the semi active friction mount for different damping characteristics is depicted. Here $F$ denotes the overall engine mount force, i.e. friction force plus force of the elastomer spring of the mount. The measurement was carried out at the eigenfrequency of the engine in the car, which is $8 \mathrm{~Hz}$. At this frequency, the damper is permanently active. The loss hysteresis can be varied from a minimum value that is determined by the damping of the elastomer spring to values larger than those of the original mount by adapting the damper characteristic. The hysteresis loops of the friction mount are very smooth and do not show at all the bumpy action of a dry friction device. The analysis of the harmonics in the measured force, displayed in the right part of Fig. 13, reveals the superior performance of the friction mount over the original mount up to 


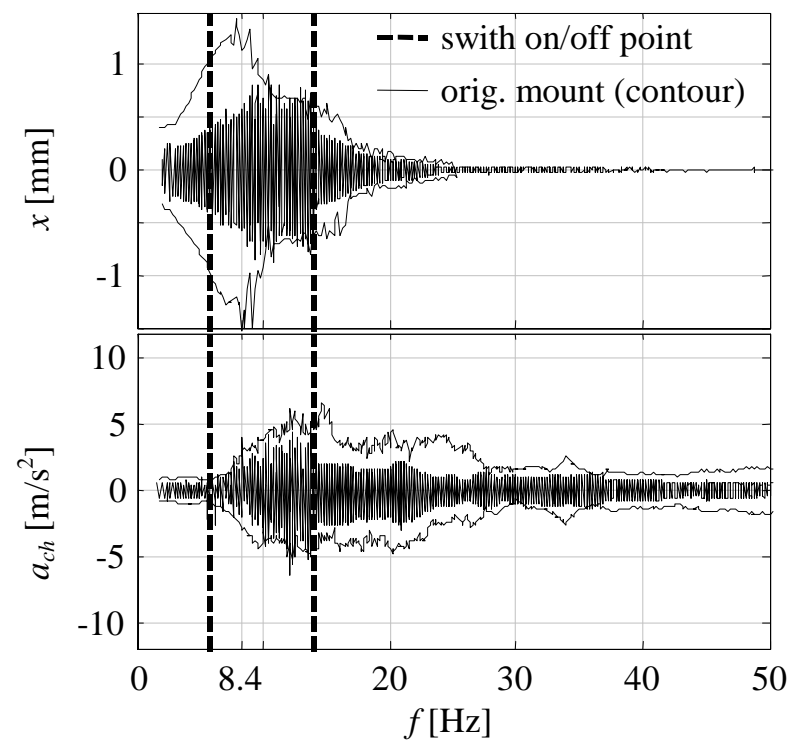

Fig. 14. Displacement of the engine mount and acceleration measured at the chassis side of the friction-mount. (comparison to the original mount).

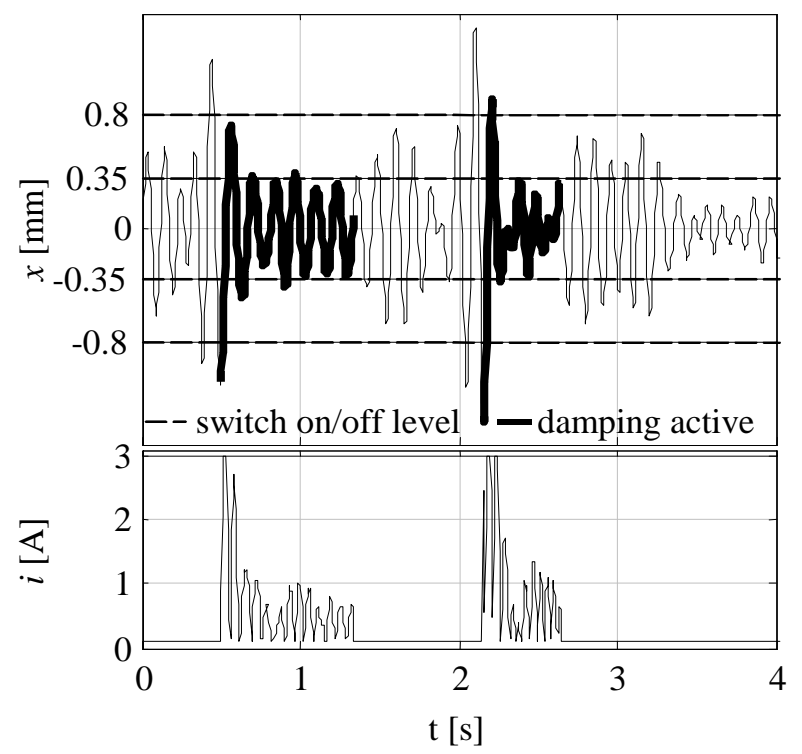

Fig. 15. Displacement of the mount and actuator current measured on a test drive on the motorway while driving over a small gap.

the 8th harmonic. This is because of the influence of the decoupler in the original (hydraulic) mount, which causes impacts at the turning points of the oscillating displacement.

Finally, the engine mount was built into the car. The front weels were excited with a sinus sweep from 1 to $50 \mathrm{~Hz}$ at a rate of $1 \mathrm{~Hz}$ per second. The displacement of the mount and the acceleration on the chassis side of the mount in direction of the displacement were measured. Figure 14 shows a frequency plot of the two quantities (which is also a time plot because of the rate of $1 \mathrm{~Hz} / \mathrm{s}$ ). The measurement of the original mount is depicted as outline for comparison purposes. While the measured displacement shows a clear resonance peak when operated with the original mount, no defined resonance peak is visible with the friction-mount. This is an advantage of the far higher damping of the friction-mount in this region. The acoustic advantage of the friction-mount is visible in the chassis acceleration, 
which is at (higher) frequencies where damping is deactivated. Figure 14 shows data measured on a test-drive on the motorway. The upper part shows the time plot of the displacement, the lower part shows the actuator current. The switch on and switch off levels for the damping function of the mount are represented by dashed lines. The measurement was recorded in a moment where the car was crossing a small gap in the road surface. One period after the displacement exceeds the switch on level of $0.8 \mathrm{~mm}$, damping is activated and a significant effect of damping can be seen. When the displacement signal remains lower than the switch off level of $0.35 \mathrm{~mm}$ for more than one period, damping is deactivated again.

\section{Conclusion}

It was shown that an electromagnetic actuator can be used, which is very light and small. The nonlinear currentforce-characteristic of the actuator is linearized. The linearization must be initialized when the device is switched on, because the actuator geometry changes with increasing wear. The initialization routine that is proposed in this paper provides precise calculation of a valid force-current-curve of the actuator. No mechanical sensor is needed because all necessary information is obtained from an electric measurement of the inductance of the coil.

The friction model proposed here is easy to identify and shows very good accordance between measured and calculated data. If deviations in the modeled friction force at direction changes are acceptable, a simplified version of the friction model can be used which does not require the small simulation time steps of the full model. In case of the semi-active friction damper, the simplified version can be used because at direction changes, the friction force shall be zero for acoustic reasons. Thus an imprecise value of the dynamic friction coefficient has no severe consequences here.

The control policy is simple but effective. Damping is activated whenever the magnitude of the eigenfrequency of the engine exceeds a predetermined level. As soon as the magnitude is lower than a certain switch off level, damping is deactivated again. When damping is active, the damper characteristic (damping force over velocity) is determined by a preselectable curve. Here the friction contact was successfully tuned to behave similar to a viscous damper.

The prototype of the friction damped engine mount proved to be superior to the original hydro mount of the test vehicle. It showed lower force levels up to the 8th harmonic, when excited with a sinusoidal displacement at the engine's natural frequency. In the car, lower acceleration levels at the chassis (when damping is off) and lower displacement magnitudes (when damping is on) could be achieved. The advantages of the semi-active friction mount level out, when permanent excitation of the engine-eigenfrequency occurs. This can be the case on very bumpy roads. On good roads, however, a clear advantage of the friction damped mount is observed.

\section{References}

[1] A. Gennesseaux, Research for New Vibration Isolation Techniques: From Hydro-Mounts to Active Mounts, Proceedings of the 1993 Noise and Vibration Conference, SAE Technical Papers No. 931324, 1993.

[2] G. Kim and R. Singh, Study of passive and adaptive hydraulic engine mount systems with emphasis on non-linear characteristics, Journal of Sound and Vibration (3) (1995), 427-453.

[3] M. Ahmadian and Y.K. Ahn, Performance analysis of magneto-rheological mounts, Journal of Intelligent Material Systems and Structures 10 (1999), 248-256.

[4] Y.K. Ahn, M. Ahmadian and S. Morishita, On the design and development of a magneto-rheological mount, Vehicle System Dynamics (Netherlands) 32 (1999), 199-216.

[5] S.B. Choi and H.J. Song, Vibration control of a passenger vehicle utilizing a semi-active ER engine mount, Vehicle System Dynamics 37 (2002), 193-216.

[6] J.S. Lane and A.A. Ferri, Friction-Induced Vibration, Chatter, Squeal and Chaos, Proceedings of the 1992 Winter Annual Meeting of The ASME DE-Vol. 49 (1992), 165-171.

[7] L. Gaul and R. Nitsche, Vibration control by interface dissipation in semi-active joints, Zeitung frr Angewandte Mathematik und Mechanik (Germany) 80(1) (2000).

[8] C.W. Stammers and T. Sireteanu, Vibration Control of Machines by use of Semi-Active Dry Friction Damping, Journal of Sound and Vibration 209(4) (1998), 671-684.

[9] C. Browler, A.J. Medland and C.W. Stammers, Vehicle vibration - active control, Journal of Measurement and Control 34 (2001), 1009.

[10] C.W. Stammers and T. Sireteanu, Control of Building Seismic Response By means of Three Semi-Active Friction Dampers, Journal of Sound and Vibration 237(5) (2000), 745-759. 

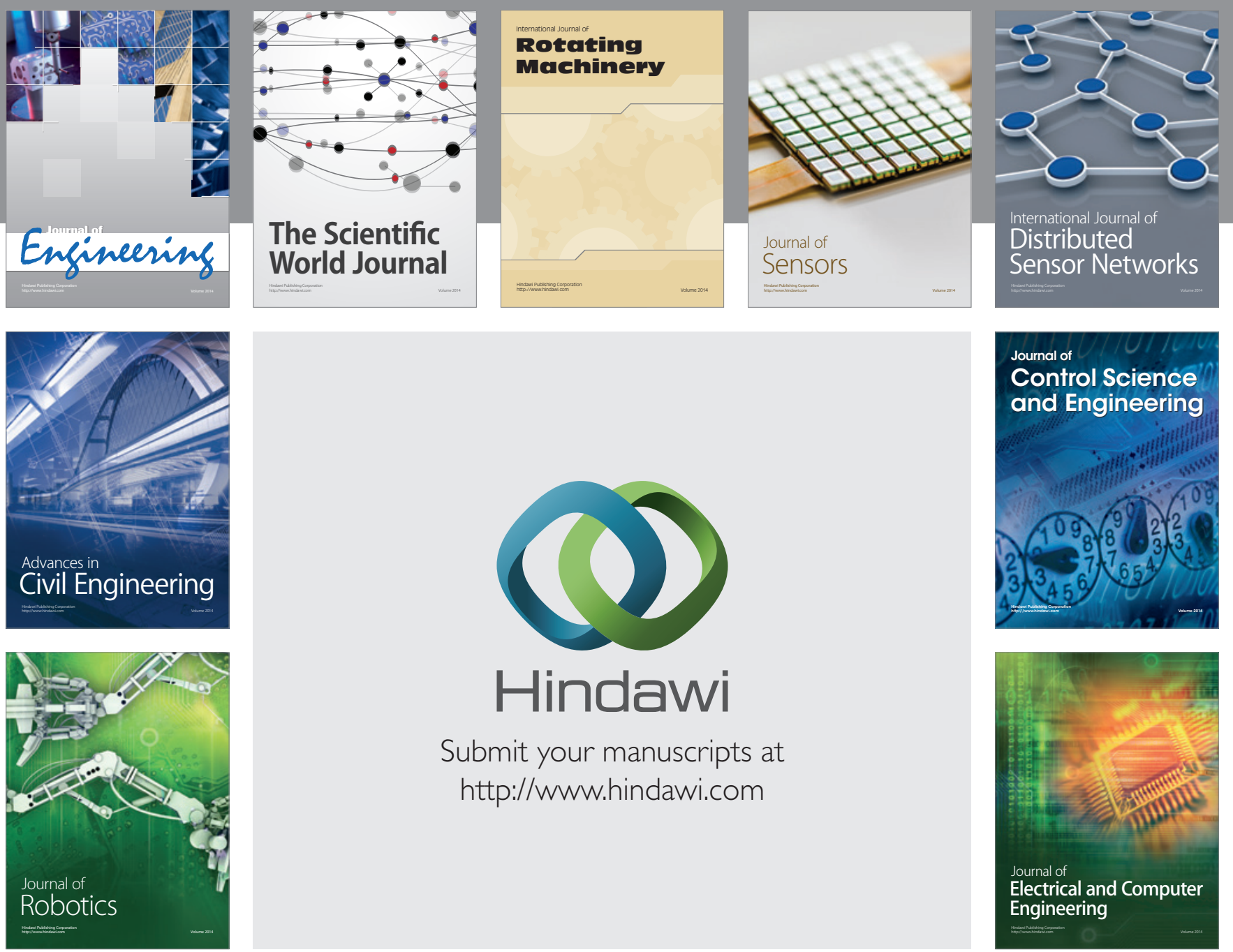

Submit your manuscripts at

http://www.hindawi.com
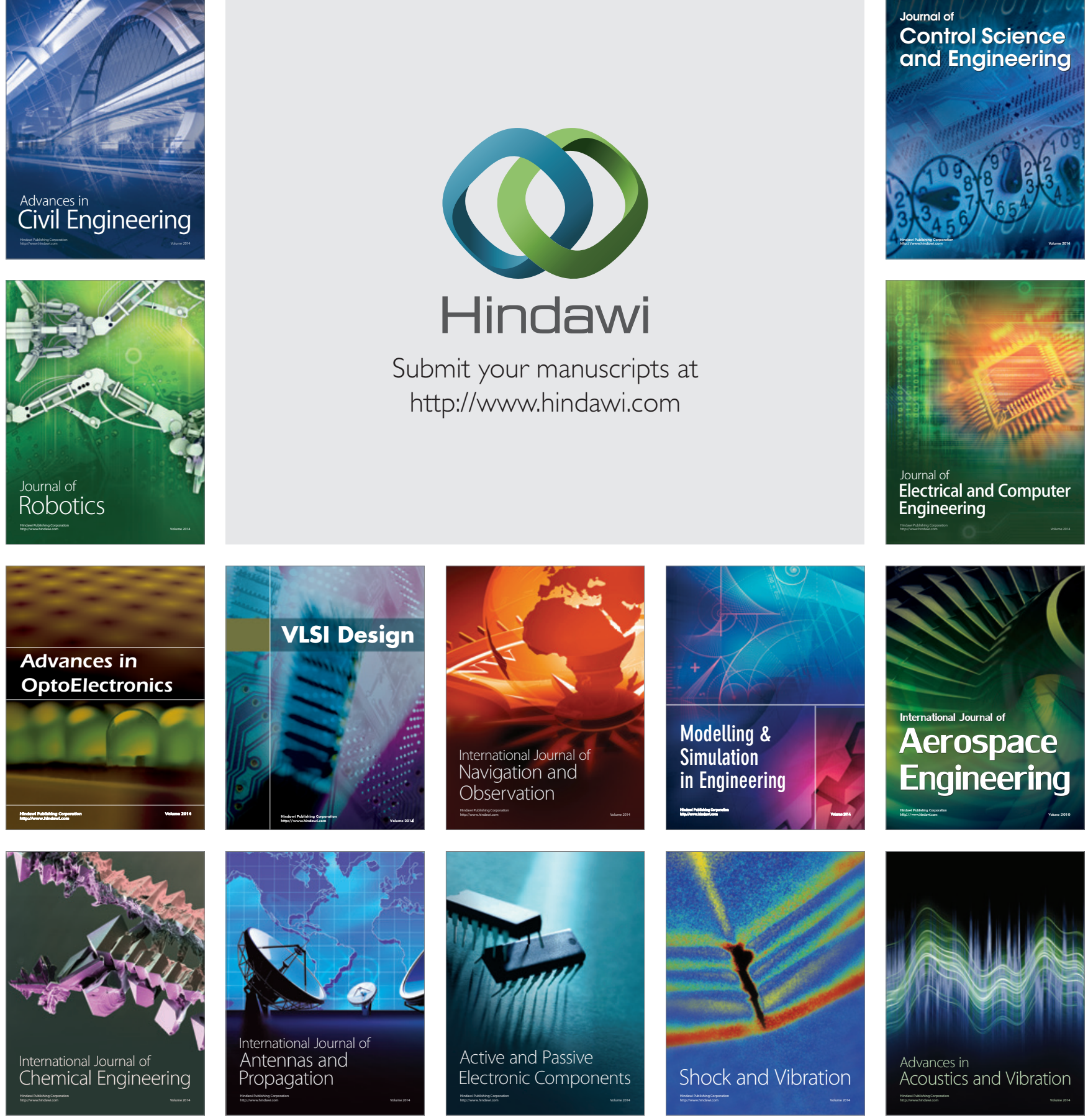